\title{
CONNECTIONS BETWEEN SINGULAR CONTROL AND OPTIMAL SWITCHING*
}

\author{
XIN GUO ${ }^{\dagger}$ AND PASCAL TOMECEK
}

\begin{abstract}
This paper builds a new theoretical connection between singular control of finite variation and optimal switching problems. This correspondence provides a novel method for solving high-dimensional singular control problems and enables us to extend the theory of reversible investment: Sufficient conditions are derived for the existence of optimal controls and for the regularity of value functions. Consequently, our regularity result links singular controls and Dynkin games through sequential optimal stopping problems.
\end{abstract}

Key words. singular stochastic control, optimal switching, Dynkin games, reversible investment

AMS subject classifications. 93E20, 49N60, 91A15, 91A55

DOI. $10.1137 / 060669024$

1. Introduction with a motivating control problem. Let $(\Omega, \mathcal{F}, P)$ be a complete probability space and $\mathbb{F}=\left\{\mathcal{F}_{t} ; 0 \leq t<\infty\right\}$ a completed filtration that is right continuous. Consider the following (motivating) singular control problem from [33]:

$$
V(x, y)=\sup _{\left(\xi^{+}, \xi^{-}\right) \in \mathcal{A}} E\left[\int_{0}^{\infty} e^{-r t} h\left(X_{t}, Y_{t}\right) d t-K^{+} \int_{0}^{\infty} e^{-r t} d \xi_{t}^{+}-K^{-} \int_{0}^{\infty} e^{-r t} d \xi_{t}^{-}\right]
$$

where $Y_{t}=y+\xi_{t}^{+}-\xi_{t}^{-},\left(\xi_{t}^{+}, \xi_{t}^{-}\right)_{t \geq 0}$ is a pair of $\mathbb{F}$-adapted, nondecreasing càglàd processes, $X_{t}$ is a diffusion process with $X_{0}=x, h\left(X_{t}, Y_{t}\right)$ is a concave function of $Y_{t}$ satisfying appropriate integrability conditions, and $K^{+}, K^{-}, r>0$ are some constants. Here the supremum is taken over the set $\mathcal{A}$ of all singular controls with a finite variation.

This multidimensional control problem and its variants have been studied extensively in both the mathematics and the economics literature. For example, taking $h$ as a concave function with a special additive form $h\left(X_{t}+Y_{t}\right)$ and $K^{-}+K^{+} \geq$ 0 , this is the well-known monotone fuel follower problem, for which explicit solutions can be found in $[4,5,6,25,24]$. In mathematical economics, (1) is a typical (ir)reversible investment problem in which a company, by adjusting its production capacity through expansion and contraction according to market fluctuations, wishes to maximize its overall expected net profit over an infinite horizon. Under the special additive form (again) of $h\left(X_{t}+Y_{t}\right)$, with $X_{t}+Y_{t}=y+\mu t+\sigma W_{t}+\xi_{t}^{+}$and $\xi_{t}^{-}=K^{-}=0$, this problem has been investigated by numerous authors (see, for instance, $[14,31,1,2,34,36,13,3,21])$. With another special form of $h\left(1-Y_{t}+X_{t} Y_{t}\right)$, where $h$ is a power function, $K^{-}=K^{+}=0$, and $Y_{t} \in[0,1]$, the problem was analyzed

\footnotetext{
${ }^{*}$ Received by the editors September 4, 2006; accepted for publication (in revised form) July 18, 2007; published electronically February 1, 2008.

http://www.siam.org/journals/sicon/47-1/66902.html

${ }^{\dagger}$ Department of Industrial Engineering and Operations Research, UC Berkeley, Berkeley, CA 94720-1777 (xinguo@ieor.berkeley.edu). This author is on leave from Cornell University.

${ }^{\ddagger}$ School of Operations Research and Industrial Engineering, Cornell University, Ithaca, NY 148533801 (pascal@orie.cornell.edu).
} 
via a dimension reduction technique in [35]. For a standard reference on irreversible investment, see [15].

Most recently, [33] treated this problem with a more general and genuinely highdimensional form, where $X_{t}$ is a geometric Brownian motion and $h$ is a function of both $X_{t}$ and $Y_{t}$, subject to some technical conditions. They used the traditional approach of the dynamic programming principle: First construct (by ad hoc methods) a solution to the Bellman equation and then validate the optimality of the solution by a sufficient verification theorem for smooth functions.

In this paper, we establish a generic theoretical connection between singular control and optimal switching problems: We define a consistency property for collections of switching controls and prove that there is an exact correspondence between the set of finite variation càglàd processes and the set of consistent collections of switching controls. We then apply this correspondence, in conjunction with direct integration arguments, to obtain an integral representation for the value function of a very general reversible investment problem in terms of the values of corresponding optimal switching problems. Finally, we exploit this representation to study the regularity of the value function and to obtain sufficient conditions on the existence of optimal controls. As a corollary, we are able to represent the value of a Dynkin game as the difference between the values of two related switching problems, thereby linking the general reversible investment problem, the Dynkin game, and the optimal switching problem.

It is worth pointing out that this approach of connecting singular control problems and related optimal stopping problems dates back to the seminal paper of [4] and has since been developed and applied to monotone singular control problems in [25, $26,27,28,29,17,18,19,2] .{ }^{1}$ Indeed, our integral representation theorem for the reversible investment problem is in part inspired by the elegant integration arguments of [2] for irreversible investment. Another closely related body of work is [11, 8, 9, 10]. However, the connections between the singular control problem, the entry-exit problem, and Dynkin's game in their works are established within the framework of forward backward stochastic differential equations and require a finite time horizon with the restrictive assumption that the control has only an additive affect on the diffusion. As such, their results cannot be used to solve the more general reversible investment problems such as (1).

Compared to all previous works and approaches, the correspondence between singular controls and switching controls in our paper does not depend on the specific form of the control problem. Thus, our methodology applies to cases for which $X_{t}$ can be any diffusion process other than the geometric Brown motion and to cases for which the running payoff function $h$ is a general and nonsmooth function of both the diffusion $X_{t}$ and the control process $Y_{t}$. In fact, our method is applicable when the underlying randomness is not necessarily captured by a diffusion. This enables us to solve very general reversible investment problems. In particular, when $h$ is smooth enough, the regularity assumptions for the value function in [33] are recovered.

The organization of the paper is as follows. In section 2 , we define consistent collections of switching controls and describe how to obtain such a collection from a singular control and vice versa. We prove that these transformations define a bijection between the set of singular controls and the set of consistent collections of switching controls and prove a change of variable formula. In section 3 , we apply this corre-

\footnotetext{
${ }^{1}$ Recently, in [22] it was observed that both the Dynkin game and the two regime optimal switching problem lead to backward stochastic differential equations with two reflecting barriers.
} 
spondence to the problem of reversible investment and show how the value function of the singular control problem can be represented in terms of the value functions of optimal switching problems. Using this representation, we prove the differentiability of the value function and show that, due to the relationship between optimal switching problems and Dynkin games, the derivative can be represented in terms of either one. Last, we give a two-dimensional example with an explicit solution.

2. Correspondence between singular controls and switching controls. In this section, we establish by explicit construction a bijection between admissible singular controls and consistent collections of switching controls with two regimes. Our result is analogous to the well-known correspondence between a nondecreasing, $\mathbb{F}$ adapted, càglàd singular control $\left(\xi_{t}\right)_{t \geq 0}$ and a collection of stopping times $\left(\tau^{\xi}(z)\right)_{z \in \mathbb{R}}$, given by

$$
\tau^{\xi}(z)=\inf \left\{t \geq 0: \xi_{t}>z\right\}, \quad \text { and } \quad \xi_{t}=\sup \left\{z \in \mathbb{R}: \tau^{\xi}(z)<t\right\} .
$$

2.1. Definitions. Let $(\Omega, \mathcal{F}, P)$ be a complete probability space and $\mathbb{F}=\left\{\mathcal{F}_{t}\right.$; $0 \leq t<\infty\}$ a filtration satisfying the usual hypotheses. Let $\mathcal{I} \subset \mathbb{R}$ be an open (possibly unbounded) interval and $\overline{\mathcal{I}}$ be its closure.

Let us first recall the notion of admissible singular controls.

DeFINITION 2.1. Given $y \in \overline{\mathcal{I}}$, an admissible singular control is a pair $\left(\xi_{t}^{+}, \xi_{t}^{-}\right)_{t \geq 0}$ of $\mathbb{F}$-adapted, nondecreasing càglàd processes such that $\xi^{+}(0)=\xi^{-}(0)=0, Y_{t}:=$ $y+\xi_{t}^{+}-\xi_{t}^{-} \in \overline{\mathcal{I}}$, for all $t \in[0, \infty)$, and $d \xi^{+}, d \xi^{-}$are supported on disjoint subsets.

We denote here $\mathcal{A}_{y}$ to be the set of admissible strategies corresponding to an initial capacity level of $y$.

Since $d \xi^{+}$and $d \xi^{-}$are supported on disjoint subsets, $\xi^{+}$and $\xi^{-}$are the positive and negative variations of $Y$, respectively. By the uniqueness of the variation decomposition, there is a one-to-one correspondence between strategies $\left(\xi^{+}, \xi^{-}\right) \in \mathcal{A}_{y}$ and $\mathbb{F}$-adapted càglàd finite variation processes $Y$, with $Y_{0}=y$ and $Y_{t} \in \overline{\mathcal{I}}$ for all $t$.

Throughout the paper, $\left(Y_{t}\right)_{t \geq 0}$ is a finite variation control process with $Y_{0}=y$.

Next, we introduce admissible switching controls (with two regimes).

Definition 2.2. A switching control $\alpha=\left(\tau_{n}, \kappa_{n}\right)_{n \geq 0}$ consists of an increasing sequence of stopping times $\left(\tau_{n}\right)_{n \geq 0}$ and a sequence of new regime values $\left(\kappa_{n}\right)_{n \geq 0}$ that are assumed immediately after each stopping time.

When there are only two distinct regimes, an optimal switching problem is often referred to as the starting and stopping problem $([12,23]$, etc.) or the entry and exit problem ([9, 16], etc.). Following convention, we label the two regimes 0 and 1.

DeFINITION 2.3. A switching control $\alpha=\left(\tau_{n}, \kappa_{n}\right)_{n \geq 0}$ is admissible if the following hold almost surely: $\tau_{0}=0, \tau_{n+1}>\tau_{n}$ for $n \geq 1, \tau_{n} \rightarrow \infty$, and for all $n \geq 0$, $\kappa_{n} \in\{0,1\}$ is $\mathcal{F}_{\tau_{n}}$ measurable, with $\kappa_{n}=\kappa_{0}$ for even $n$ and $\kappa_{n}=1-\kappa_{0}$ for odd $n$.

Alternatively, an admissible switching control has a more mathematically convenient representation given by its regime indicator function.

Proposition 2.4. There is a one-to-one correspondence between admissible switching controls and the regime indicator function $I_{t}(\omega)$, which is an $\mathbb{F}$-adapted càglàd process of finite variation, so that $I_{t}(\omega): \Omega \times[0, \infty) \rightarrow\{0,1\}$, with

$$
I_{t}:=\sum_{n=0}^{\infty} \kappa_{n} 1_{\left\{\tau_{n}<t \leq \tau_{n+1}\right\}}, \quad I_{0}=\kappa_{0} .
$$

Copyright (C) by SIAM. Unauthorized reproduction of this article is prohibited. 
Lemma 2.5. Given an admissible switching control $\alpha=\left(\tau_{n}, \kappa_{n}\right)_{n \geq 0}$, define the increasing càglàd processes $I^{+}$and $I^{-}$by

$$
I_{t}^{+}:=\sum_{n>0, \kappa_{n}=1}^{\infty} 1_{\left\{\tau_{n}<t\right\}}, \quad I_{0}^{+}=0 \quad \text { and } \quad I_{t}^{-}:=\sum_{n>0, \kappa_{n}=0}^{\infty} 1_{\left\{\tau_{n}<t\right\}}, \quad I_{0}^{-}=0 .
$$

Then for all $t \geq 0, I_{t}^{ \pm}<\infty$ almost surely, $I_{t}=\kappa_{0}+I_{t}^{+}-I_{t}^{-}$, and $I_{t}^{+}\left(I_{t}^{-}\right)$is the positive (negative) variation of the corresponding regime indicator function.

Finally, we define a class of consistent collections of switching controls. We shall see later that it is exactly this class of consistent collections of switching controls that corresponds to singular controls of finite variation.

Definition 2.6. Let $y \in \overline{\mathcal{I}}$ be given, and for each $z \in \mathcal{I}$, let $\alpha(z)=\left(\tau_{n}(z)\right.$, $\left.\kappa_{n}(z)\right)_{n \geq 0}$ be a switching control. The collection $(\alpha(z))_{z \in \mathcal{I}}$ is consistent if

$$
\begin{aligned}
& \alpha(z) \text { is admissible for Lebesgue-almost every } z \in \mathcal{I}, \\
& I_{0}(z):=\kappa_{0}(z)=1_{\{z \leq y\}} \text { for Lebesgue-almost every } z \in \mathcal{I},
\end{aligned}
$$

and, for all $t<\infty$,

$$
\begin{aligned}
& \int_{\mathcal{I}}\left(I_{t}^{+}(z)+I_{t}^{-}(z)\right) d z<\infty, \text { almost surely, and } \\
& I_{t}(z) \text { is decreasing in } z \text { for } \mathbb{P} \otimes d z \text {-almost every }(\omega, z) .
\end{aligned}
$$

Here $I_{t}(z), I_{t}^{+}(z)$, and $I_{t}^{-}(z)$ are defined as in (2) and Lemma 2.5 .

For $I_{t}(z)$ to be decreasing in $z$ for $\mathbb{P} \otimes d z$-almost every $(\omega, z)$, it means there exists a set $E \subset \Omega \times \overline{\mathcal{I}}$ such that $\mathbb{P} \otimes d z(E)=0$, and if $\left(\omega, z_{0}\right),\left(\omega, z_{1}\right) \in(\Omega \times \overline{\mathcal{I}}) \backslash E$, with $z_{0} \leq z_{1}$, then $I_{t}\left(\omega, z_{0}\right) \geq I_{t}\left(\omega, z_{1}\right)$.

2.2. Bijection. First, we describe how a consistent collection of switching controls can be obtained from an admissible singular control. To this end, we quote two technical lemmas, the first one adapted from [20, Theorem 5.5.1].

Lemma 2.7 (Evans and Gariepy). Let $f:[0, \infty) \rightarrow \mathbb{R}$ be a function of finite (i.e., locally bounded) variation, and define $E:[0, \infty) \times \mathbb{R} \rightarrow \mathbb{R}$ by $E(s, z)=1_{\{f(s)>z\}}$. Then

1. the function $E(\cdot, z)$ is of finite variation for almost all $z \in \mathbb{R}$, and

2. $\|d f\|([0, t))=\int_{-\infty}^{\infty}\|d E(\cdot, z)\|([0, t)) d z$ for all $t \in(0, \infty)$.

Lemma 2.8. Let $f:[0, \infty) \rightarrow\{0,1\}$ be of finite variation, and define $g(t)=$ $\lim _{s \uparrow t} f(s)$. Then almost surely the paths of $g$ are càglàd, and, for all $T<\infty$,

$$
\|d g\|([0, T)) \leq\|d f\|([0, T))<\infty
$$

Proposition 2.9 (from singular to switching controls). Given $\left(\xi^{+}, \xi^{-}\right) \in \mathcal{A}_{y}$, define a switching control $\alpha(z)=\left(\tau_{n}(z), \kappa_{n}(z)\right)_{n \geq 0}$ for each $z \in \mathcal{I}$ through the regime indicator function $I_{t}(z):=\lim _{s \uparrow t} 1_{\left\{Y_{s}>z\right\}}$. Then the resulting collection $(\alpha(z))_{z \in \mathcal{I}}$ of switching controls is consistent.

Proof. First we show that $I$ is a regime indicator function as per Proposition 2.4. Let $Y_{t}=y+\xi_{t}^{+}-\xi_{t}^{-}$. Since $Y$ is $\mathbb{F}$-adapted, so is $I$. $(z)$. Furthermore, since $Y$ is of finite variation, Lemma 2.7 implies that the function $s \rightarrow 1_{\left\{Y_{s}(\omega)>z\right\}}$ is of finite variation for $\mathbb{P} \otimes d z$-almost every $(\omega, z)$. Hence $I_{t}(\omega, z)$ is càglàd and of finite variation $(\omega, z)$-a.e.

Copyright $@$ by SIAM. Unauthorized reproduction of this article is prohibited. 
by Lemma 2.8. Thus, for almost all $z$, there is an admissible switching control $\alpha(z)$ corresponding to $I(z)$.

Moreover, Lemma 2.7, in conjunction with Lemmas 2.8 and 2.5, implies that

$$
\begin{aligned}
\|d Y \cdot\|([0, t))=\xi_{t}^{+}+\xi_{t}^{-} & =\int_{-\infty}^{\infty}\left\|d 1_{\{Y .>z\}}\right\|([0, t)) d z \geq \int_{\mathcal{I}}\|d I .(z)\|([0, t)) d z \\
& =\int_{\mathcal{I}} I_{t}^{+}(z)+I_{t}^{-}(z) d z .
\end{aligned}
$$

Hence $\int_{\mathcal{I}} I_{t}^{+}(z)+I_{t}^{-}(z) d z<\infty$. In addition, $I_{t}(z)$ is decreasing in $z$ and $I_{0}(z)=1_{\{y \geq z\}}$ for all $z$ (except for $z=y$ ), so the collection $(\alpha(z))_{z \in \mathcal{I}}$ is consistent.

Next, we construct an admissible singular control $\left(\xi^{+}, \xi^{-}\right)$from a consistent collection of switching controls via their regime indicator functions. Consequently, we give two useful representations of a finite variation process $Y$.

Proposition 2.10 (from switching controls to singular controls). Given $y \in \overline{\mathcal{I}}$ and a consistent collection of switching controls $(\alpha(z))_{z \in \mathcal{I}}$, define two processes $\xi^{+}$and $\xi^{-}$by setting $\xi_{0}^{+}=0, \xi_{0}^{-}=0$, and for $t>0: \xi_{t}^{+}:=\int_{\mathcal{I}} I_{t}^{+}(z) d z, \quad \xi_{t}^{-}:=\int_{\mathcal{I}} I_{t}^{-}(z) d z$. Then

1. the pair $\left(\xi^{+}, \xi^{-}\right) \in \mathcal{A}_{y}$ is an admissible singular control,

2. up to indistinguishability,

$$
Y_{t}=y+\int_{y}^{\infty} I_{t}(z) 1_{\{z \in \mathcal{I}\}} d z+\int_{-\infty}^{y}\left(I_{t}(z)-1\right) 1_{\{z \in \mathcal{I}\}} d z, \quad \text { and }
$$

3. for all $t$, we almost surely have

$$
Y_{t}=\operatorname{ess} \sup \left\{z \in \mathcal{I}: I_{t}(z)=1\right\}=\operatorname{ess} \inf \left\{z \in \mathcal{I}: I_{t}(z)=0\right\}
$$

where $\operatorname{ess} \sup \emptyset:=\inf \mathcal{I}$ and $\operatorname{ess} \inf \emptyset:=\sup \mathcal{I}$.

Proof.

1. The proof is obvious from the property of $(\alpha(z))_{z \in \mathcal{I}}$, Definition 2.6, and Lemma 2.5.

2. By applying Lemma 2.5, we have a.e. for every $t \geq 0$,

$$
\begin{aligned}
Y_{t} & =y+\xi_{t}^{+}-\xi_{t}^{-}=y+\int_{\mathcal{I}}\left(I_{t}^{+}(z)-I_{t}^{-}(z)\right) d z \\
& =y+\int_{y}^{\infty} I_{t}(z) 1_{\{z \in \mathcal{I}\}} d z+\int_{-\infty}^{y}\left(I_{t}(z)-1\right) 1_{\{z \in \mathcal{I}\}} d z .
\end{aligned}
$$

3. By fixing $t \geq 0$ and observing that $I_{t}(z) \in\{0,1\}$ is decreasing in $z,(\omega, z)$-a.e., we see

$$
\begin{aligned}
Y_{t} & =y+\int_{y}^{\infty} I_{t}(z) 1_{\{z \in \mathcal{I}\}} d z+\int_{-\infty}^{y}\left(I_{t}(z)-1\right) 1_{\{z \in \mathcal{I}\}} d z \\
& =y+\left[\operatorname{ess} \sup \left\{z \in \mathcal{I}: I_{t}(z)=1\right\}-y\right]^{+}-\left[\operatorname{ess} \sup \left\{z \in \mathcal{I}: I_{t}(z)=1\right\}-y\right]^{-} \\
& =\operatorname{ess} \sup \left\{z \in \mathcal{I}: I_{t}(z)=1\right\} .
\end{aligned}
$$

Copyright (c) by SIAM. Unauthorized reproduction of this article is prohibited. 
Proposition 2.11 (one-to-one mapping). The mapping from consistent collections of switching controls to singular controls defined by Proposition 2.10 is one-toone.

Finally, we shall show that the two mappings defined in Propositions 2.9 and 2.10 are inverses of each other, thus inducing a bijection.

Theorem 2.12 (bijection). The mappings in Propositions 2.9 and 2.10 define a bijection between admissible singular controls $\left(\xi^{+}, \xi^{-}\right) \in \mathcal{A}_{y}$ and consistent collections of switching controls (up to equivalence).

Proof. To show that the constructions in Propositions 2.9 and 2.10 are inverses of each other, let us start with a singular control $\left(\xi^{+}, \xi^{-}\right) \in \mathcal{A}_{y}$. First, applying Proposition 2.9 to $\left(\xi^{+}, \xi^{-}\right) \in \mathcal{A}_{y}$ generates a collection $(\alpha(z))_{z \in \mathcal{I}}$ of switching controls. Then, applying Proposition 2.10 to $(\alpha(z))_{z \in \mathcal{I}}$ yields another pair of singular controls $\left(\tilde{\xi}^{+}, \tilde{\xi}^{-}\right)$. We shall show that $\left(\xi^{+}, \xi^{-}\right)=\left(\tilde{\xi}^{+}, \tilde{\xi}^{-}\right)$.

By Proposition 2.9 we have $I_{t}(z)=\lim _{s \uparrow t} 1_{\left\{Y_{s}>z\right\}}$. Therefore, by Proposition 2.10, the dominated convergence theorem, and with $Y \in \mathcal{I}$ almost surely, we have

$$
\begin{aligned}
\tilde{Y}_{t} & =y+\int_{y}^{\infty} I_{t}(z) 1_{\{z \in \mathcal{I}\}} d z+\int_{-\infty}^{y}\left(I_{t}(z)-1\right) 1_{\{z \in \mathcal{I}\}} d z \\
& =y+\int_{y}^{\infty} \lim _{s \uparrow t} 1_{\left\{Y_{s}>z\right\}} 1_{\{z \in \mathcal{I}\}} d z-\int_{-\infty}^{y} \lim _{s \uparrow t} 1_{\left\{Y_{s} \leq z\right\}} 1_{\{z \in \mathcal{I}\}} d z \\
& =y+\lim _{s \uparrow t} \int_{y}^{\infty} 1_{\left\{Y_{s}>z\right\}} 1_{\{z \in \mathcal{I}\}} d z-\lim _{s \uparrow t} \int_{-\infty}^{y} 1_{\left\{Y_{s} \leq z\right\}} 1_{\{z \in \mathcal{I}\}} d z \\
& =y+\left[Y_{t}-y\right]^{+}-\left[Y_{t}-y\right]^{-}=Y_{t} .
\end{aligned}
$$

Thus, $\tilde{Y}_{t}$ and $Y_{t}$ have the same variation decompositions, and hence $\left(\xi^{+}, \xi^{-}\right)=\left(\tilde{\xi}^{+}\right.$, $\left.\tilde{\xi}^{-}\right)$almost surely.

Since the mapping in Proposition 2.10 is one-to-one by Proposition 2.11, this proves that the mappings in Propositions 2.10 and 2.9 are inverses of each other, and hence a bijection exists.

Given this correspondence, we shall use the following terminology in what follows. Given a singular control $\left(\xi^{+}, \xi^{-}\right) \in \mathcal{A}_{y}$, the corresponding collection of switching controls $(\alpha(z))_{z \in \mathcal{I}}$ refers to the one defined in Proposition 2.9; given a consistent collection of switching controls, the corresponding singular control refers to that in Proposition 2.10.

2.3. Change of variable formula. With the bijection established in Theorem 2.12 , we are ready to establish a change of variable formula for integration with respect to the variation of a singular control.

Lemma 2.13. Let $\left(\xi^{+}, \xi^{-}\right) \in \mathcal{A}_{y}$ be an admissible singular control and $(\alpha(z))_{z \in \mathcal{I}}$ be the corresponding collection of switching controls. For every càdlàg process $g$ : $\Omega \times[0, \infty] \rightarrow[0, \infty)$, with $g(\infty) \equiv 0$,

$$
\begin{aligned}
\int_{[0, \infty)} g(t) d \xi_{t}^{+} & =\int_{\mathcal{I}} \sum_{\substack{n>0 \\
\kappa n=1}} g\left(\tau_{n}(z)\right) d z, \quad \text { a.s. }, \\
\text { and } \int_{[0, \infty)} g(t) d \xi_{t}^{-} & =\int_{\mathcal{I}} \sum_{\substack{n>0 \\
\kappa_{n}=0}} g\left(\tau_{n}(z)\right) d z, \quad \text { a.s. }
\end{aligned}
$$

Copyright (c) by SIAM. Unauthorized reproduction of this article is prohibited. 
Proof. We shall show only the result for $\xi^{+}$as the proof for $\xi^{-}$is almost identical. Suppose $g$ is a càdlàg process with the representation

$$
g(t)=\sum_{i=0}^{N} g_{i} 1_{\left[\sigma_{i}, \sigma_{i+1}\right)}(t),
$$

where $N$ is finite and constant, $0=\sigma_{0} \leq \sigma_{1} \leq \cdots \leq \sigma_{N+1}<\infty, g_{i} \in \mathcal{F}_{\sigma_{i}}$, and $\left|g_{i}\right|<$ $\infty$ almost surely. Then, by Proposition 2.10 , the monotone convergence theorem, and Fubini's theorem,

$$
\begin{aligned}
\int_{[0, \infty)} g(t) d \xi_{t}^{+} & =\int_{[0, \infty)} \sum_{i=0}^{N} g_{i} 1_{\left[\sigma_{i}, \sigma_{i+1}\right)}(t) d \xi_{t}^{+}=\sum_{i=0}^{N} g_{i} \int_{[0, \infty)} 1_{\left[\sigma_{i}, \sigma_{i+1}\right)}(t) d \xi_{t}^{+} \\
& =\sum_{i=0}^{N} g_{i}\left(\xi_{\sigma_{i+1}}^{+}-\xi_{\sigma_{i}}^{+}\right) \\
& =\sum_{i=0}^{N} g_{i}\left(\int_{\mathcal{I}} \sum_{\substack{n>0 \\
\kappa_{n}=1}} 1_{\left\{\tau_{n}(z)<\sigma_{i+1}\right\}} d z-\int_{\mathcal{I}} \sum_{\substack{n>0 \\
\kappa_{n}=1}} 1_{\left\{\tau_{n}(z)<\sigma_{i}\right\}} d z\right) \\
& =\sum_{i=0}^{N} g_{i}\left(\int_{\mathcal{I}} \sum_{\substack{n>0 \\
\kappa n=1}} 1_{\left\{\sigma_{i} \leq \tau_{n}(z)<\sigma_{i+1}\right\}} d z\right) \\
& =\int_{\mathcal{I}} \sum_{\substack{n>0 \\
\kappa_{n}=1}}^{\infty} \sum_{i=0}^{N} g_{i} 1_{\left\{\sigma_{i} \leq \tau_{n}(z)<\sigma_{i+1}\right\}} d z \\
& =\int_{\mathcal{I}} \sum_{\substack{n>0 \\
\kappa n=1}} g\left(\tau_{n}(z)\right) d z .
\end{aligned}
$$

Since piecewise constant left continuous functions can uniformly approximate càglàd functions, this formula holds for all càglàd processes.

In particular, when $Y$ is nondecreasing (i.e., $\xi^{-} \equiv 0$ ), $\overline{\mathcal{I}}=[0, \infty)$, and $y \geq 0$, we have $\tau_{n}(z) \equiv 0$ for all $n>1$ and for $n=1$ when $z \leq y$. In this case, our change of variable formula reduces to the one for monotone controls in [2], after adjusting for notational differences:

$$
\int_{[0, \infty)} g(t) d \xi_{t}^{+}=\int_{y}^{\infty} g\left(\tau_{1}(z)\right) d z .
$$

3. Application: Reversible investment. Having established the correspondence between singular controls and consistent collections of switching controls, we shall illustrate how this theory can be applied to solving singular control problems.

As an example, we return to the aforementioned infinite-horizon, reversible investment problem (1): A company adjusts its reversible production capacity (or investment) level by proper controls of expansion and contraction in the presence of a stochastic economic environment. The net profit of such an investment depends on the running production function of the actual capacity, the economic uncertainty 
such as price or demand for the product, the benefits of contraction (e.g., via spinning off part of the business), and the cost of expanding and reducing the capital. The company's objective is to maximize the expected profit over an infinite time horizon by controlling expansion and contraction.

3.1. The singular control problem for reversible investment. More specifically, the instantaneous operating profit of the company is a function of the production capacity and random variables representing the uncertain economic environment:

$$
\Pi(\omega, t, z): \Omega \times[0, \infty) \times \overline{\mathcal{I}} \rightarrow \mathbb{R} .
$$

The unit cost of increasing the capacity at time $t$ is $\gamma_{+}(\omega, t): \Omega \times[0, \infty) \rightarrow \mathbb{R}$, and the unit cost of decreasing capacity is $\gamma_{-}(\omega, t): \Omega \times[0, \infty) \rightarrow \mathbb{R}$, where both $\gamma_{+}$and $\gamma_{-}$are adapted to $\mathbb{F} .^{2}$

The control of the production capacity $Y_{t}$ is represented by a pair $\left(\xi_{t}^{+}, \xi_{t}^{-}\right)_{t \geq 0}$ of $\mathbb{F}$-adapted, nondecreasing càglàd processes such that

$$
\begin{aligned}
& \xi^{+}(0)=\xi^{-}(0)=0, \\
& Y_{t}=y+\xi_{t}^{+}-\xi_{t}^{-} \in \overline{\mathcal{I}} \quad \forall t \in[0, \infty) .
\end{aligned}
$$

Here $\xi_{t}^{+}$and $\xi_{t}^{-}$represent the cumulative expansion and reduction of capital until time $t$, respectively. We say the policy $\left(\xi^{+}, \xi^{-}\right)$is integrable if the integrability condition is satisfied for the initial capacity level $y$. That is,

$$
\mathbb{E}\left[\int_{0}^{\infty}\left|\Pi\left(t, Y_{t}\right)\right| d t+\int_{[0, \infty)}\left|\gamma_{+}(t)\right| d \xi_{t}^{+}+\int_{[0, \infty)}\left|\gamma_{-}(t)\right| d \xi_{t}^{-}\right]<\infty .
$$

We denote $\mathcal{A}_{y}^{\prime} \subset \mathcal{A}_{y}$ as the set of integrable strategies.

Faced with these profit and cost functions, the company must choose an investment strategy of capacity expansion and reduction which produces the following expected payoff over an infinite horizon:

$$
J\left(y, \xi^{+}, \xi^{-}\right):=\mathbb{E}\left[\int_{0}^{\infty} \Pi\left(t, Y_{t}\right) d t-\int_{[0, \infty)} \gamma_{+}(t) d \xi_{t}^{+}-\int_{[0, \infty)} \gamma_{-}(t) d \xi_{t}^{-}\right] .
$$

The objective is to maximize over all integrable policies $\left(\xi^{+}, \xi^{-}\right) \in \mathcal{A}_{y}^{\prime}$. Accordingly, the value function is defined as

$$
V(y):=\sup _{\left(\xi^{+}, \xi^{-}\right) \in \mathcal{A}_{y}^{\prime}} J\left(y, \xi^{+}, \xi^{-}\right) .
$$

Note that, for any $y \in \overline{\mathcal{I}}, \mathcal{A}_{y}^{\prime}$ is not empty, as the expected profit of not investing at all (i.e., $\xi^{+} \equiv 0 \equiv \xi^{-}$) is finite and is given by

$$
R(y):=J(y, 0,0)=\mathbb{E}\left[\int_{0}^{\infty} \Pi(t, y) d t\right] .
$$

Throughout the remaining section, we impose several conditions.

\footnotetext{
${ }^{2}$ When there is no risk of ambiguity, we suppress the dependence of the profit and cost functions on $\omega$, writing $\Pi(t, z), \gamma_{+}(t)$, and $\gamma_{-}(t)$.
} 


\section{Standing assumptions.}

A1. $\Pi$ is concave in $y$ and continuous at the boundary of $\mathcal{I}$, so that for $y_{1}<$ $y_{2} \in \overline{\mathcal{I}}$,

$$
\Pi\left(t, y_{2}\right)-\Pi\left(t, y_{1}\right):=\int_{y_{1}}^{y_{2}} \pi(t, z) d z
$$

where $\pi$ is decreasing in $z$ a.s. and adapted to $\mathbb{F}$. Furthermore,

$$
\begin{aligned}
& \mathbb{E}\left[\int_{0}^{\infty}|\Pi(t, z)| d t\right]<\infty \quad \forall z \in \overline{\mathcal{I}}, \\
& \mathbb{E}\left[\int_{0}^{\infty}|\pi(t, z)| d t\right]<\infty \quad \forall z \in \mathcal{I} .
\end{aligned}
$$

This assumption implies that the value function is well-defined, and, although it may take values of $+\infty$, it is never $-\infty$ since $V(y) \geq R(y)>-\infty$ by (15).

A2. $\gamma_{+}$and $\gamma_{-}$are adapted to $\mathbb{F}, \gamma^{ \pm}(\infty):=0$ and

$$
\gamma_{+}(t)+\gamma_{-}(t)>0, \quad \text { for all } t, \text { a.s. }
$$

This restriction eliminates the opportunity of making profit by simply switching regimes and immediately switching back.

A3. - If $\mathcal{I}$ is not bounded above, then $\gamma_{+}(t) \geq 0$ for all $t$ almost surely;

- if $\mathcal{I}$ is not bounded below, $\gamma_{-}(t) \geq 0$ for all $t$ almost surely.

This is to ensure that, when the domain is unbounded, an arbitrarily large profit is not obtainable by arbitrarily large changes in the capacity level.

A very special case for the above problem $(12)$ is $\Pi(\omega, t, z)=e^{-\rho t}\left(X_{t}^{x}(\omega)\right)^{\lambda} z^{\beta}$, where the randomness in the economy is captured by the price process $X$ of the commodity, and $X$ is modeled by a geometric Brownian motion $d X_{t}^{x}=b X_{s}^{x} d t+$ $\sqrt{2} \sigma X_{s}^{x} d W_{s}$, with $X_{0}=x>0$. The cost functions are $\gamma_{+}(\omega, t)=e^{-\rho t} K_{1}, \gamma_{-}(\omega, t)=$ $e^{-\rho t} K_{0}$ for some constant $\rho>0, K_{0}, K_{1}$. We shall provide a detailed analysis and an explicit solution to this case in section 3.4.

3.2. The corresponding optimal switching problems. The key to using the connection between singular controls and switching controls to solve problem (12) in section 3.1 is to write the payoff of this problem in terms of the payoffs of its corresponding optimal switching problems. This is accomplished by exploiting the absolute continuity of the running payoff and the change of variable formula for the cost processes.

3.2.1. Switching controls from singular controls. First, given the running profit and cost functions from the singular control problem (12), we define a collection of optimal switching problems, indexed by $z \in \mathcal{I}$.

Definition 3.1. The switching cost process $\gamma: \Omega \times[0, \infty) \times\{0,1\} \rightarrow \mathbb{R}$ is given by

$$
\gamma(t, \kappa):=\gamma_{+}(t) 1_{\{\kappa=1\}}+\gamma_{-}(t) 1_{\{\kappa=0\}} .
$$

Here $\gamma(t, \kappa)$ represents the cost of switching to regime $\kappa$ at time $t$.

The following lemma shows that, for the integrable singular control $\left(\xi^{+}, \xi^{-}\right) \in \mathcal{A}_{y}^{\prime}$, the switching controls in the corresponding collection satisfy a certain integrability 
condition. It is a simple application of Fubini's theorem, from Lemma 2.13 and condition (10).

Lemma 3.2. If $\left(\xi^{+}, \xi^{-}\right) \in \mathcal{A}_{y}^{\prime}$, then, for the corresponding consistent collection of switching controls $(\alpha(z))_{z \in \mathcal{I}}$, we have $\alpha(z) \in \mathcal{B}$ for Lebesgue almost every $z \in \mathcal{I}$, where $\mathcal{B}$ is the set of admissible switching controls $\left(\tau_{n}, \kappa_{n}\right)_{n \geq 0}$ satisfying

$$
\mathbb{E}\left[\sum_{n=1}^{\infty}\left|\gamma\left(\tau_{n}, \kappa_{n}\right)\right|<\infty .\right.
$$

Note that the converse of the lemma is not true: A consistent collection of switching controls, each of which is integrable, does not necessarily correspond to an integrable singular control.

Next, we establish the following.

Proposition 3.3. Assume $\left(\xi^{+}, \xi^{-}\right) \in \mathcal{A}_{y}^{\prime}$. Let $(\alpha(z))_{z \in \mathcal{I}}$ be the corresponding consistent collection of switching controls with regime indicator functions $I(z)$; then

$$
J\left(y, \xi^{+}, \xi^{-}\right)-R(y)=\int_{y}^{\infty} m_{+}(z, \alpha(z)) 1_{\{z \in \mathcal{I}\}} d z+\int_{-\infty}^{y} m_{-}(z, \alpha(z)) 1_{\{z \in \mathcal{I}\}} d z,
$$

where

$$
\begin{aligned}
m_{+}(z, \alpha) & :=\mathbb{E}\left[\int_{0}^{\infty} \pi(t, z) I_{t} d t-\sum_{n=1}^{\infty} \gamma\left(\tau_{n}, \kappa_{n}\right)\right] \in(-\infty, \infty) \\
\text { and } m_{-}(z, \alpha) & :=\mathbb{E}\left[\int_{0}^{\infty}-\pi(t, z)\left(1-I_{t}\right) d t-\sum_{n=1}^{\infty} \gamma\left(\tau_{n}, \kappa_{n}\right)\right] \in(-\infty, \infty) .
\end{aligned}
$$

Here $m_{+}(z, \alpha)$ and $m_{-}(z, \alpha)$ are two expected payoffs for the switching controls for each $z \in \mathcal{I}$ and $\alpha \in \mathcal{B}$, with $\kappa_{0}=k \in\{0,1\}$.

Proof of Proposition 3.3. Since $\left(\xi^{+}, \xi^{-}\right) \in \mathcal{A}_{y}^{\prime}$, we have the integrability conditions (10) and (18). By applying Lemma 2.13 to the positive and negative parts of $\gamma_{+}$and $\gamma_{-}$, we see that

$$
\int_{[0, \infty)} \gamma_{+}(t) d \xi_{t}^{+}+\int_{[0, \infty)} \gamma_{-}(t) d \xi_{t}^{-}=\int_{-\infty}^{\infty} \sum_{n=1}^{\infty} \gamma\left(\tau_{n}(z), \kappa_{n}(z)\right) d z .
$$

Moreover, Proposition 2.9 implies that $I_{t}(z)=\lim _{s \uparrow t} 1_{\left\{Y_{s}>z\right\}},(\omega, z)$-a.e. and that $I .(z)$ is of finite variation, $(\omega, z)$-a.e. Thus, $I_{t}(z)=1_{\left\{Y_{t}>z\right\}},(\omega, z, t)$-a.e.

Therefore, by Fubini's theorem and (14), we almost surely have

$$
\begin{aligned}
\int_{0}^{\infty}\left(\Pi\left(t, Y_{t}\right)-\Pi(t, y)\right) d t= & \int_{0}^{\infty} \int_{y}^{\infty} \pi(t, z) 1_{\left\{Y_{t}>z\right\}} d z d t \\
& -\int_{0}^{\infty} \int_{-\infty}^{y} \pi(t, z) 1_{\left\{Y_{t} \leq z\right\}} d z d t \\
= & \int_{y}^{\infty} \int_{0}^{\infty} \pi(t, z) I_{t}(z) d t d z \\
& +\int_{-\infty}^{y} \int_{0}^{\infty}-\pi(t, z)\left(1-I_{t}(z)\right) d t d z
\end{aligned}
$$

Copyright (c) by SIAM. Unauthorized reproduction of this article is prohibited. 
Resorting again to the integrability conditions (10) and (18) and Fubini's theorem yields

$$
\begin{aligned}
J\left(y, \xi^{+}, \xi^{-}\right)-R(y) & \\
= & \mathbb{E}\left[\int_{0}^{\infty} \Pi\left(t, Y_{t}\right) d t-\int_{[0, \infty)} \gamma_{+}(t) d \xi_{t}^{+}-\int_{[0, \infty)} \gamma_{-}(t) d \xi_{t}^{-}\right]-\mathbb{E}\left[\int_{0}^{\infty} \Pi(t, y) d t\right] \\
= & \mathbb{E}\left[\int_{y}^{\infty} \int_{0}^{\infty} \pi(t, z) I_{t}(z) d t-\sum_{n=1}^{\infty} \gamma\left(\tau_{n}(z), \kappa_{n}(z)\right) d z\right] \\
& +\mathbb{E}\left[\int_{-\infty}^{y} \int_{0}^{\infty}-\pi(t, z)\left(1-I_{t}(z)\right) d t-\sum_{n=1}^{\infty} \gamma\left(\tau_{n}(z), \kappa_{n}(z)\right) d z\right] \\
= & \int_{y}^{\infty} \mathbb{E}\left[\int_{0}^{\infty} \pi(t, z) I_{t}(z) d t-\sum_{n=1}^{\infty} \gamma\left(\tau_{n}(z), \kappa_{n}(z)\right)\right] d z \\
& +\int_{-\infty}^{y} \mathbb{E}\left[\int_{0}^{\infty}-\pi(t, z)\left(1-I_{t}(z)\right) d t-\sum_{n=1}^{\infty} \gamma\left(\tau_{n}(z), \kappa_{n}(z)\right)\right] d z \\
= & \int_{y}^{\infty} m_{+}(z ; \alpha(z)) d z+\int_{-\infty}^{y} m_{-}(z ; \alpha(z)) d z .
\end{aligned}
$$

The finiteness of the payoff for $z \in \mathcal{I}$ follows from the assumed integrability of $\pi$ in (16) and $\left|I_{t}\right| \leq 1$.

3.2.2. Representation theorem. Now, for each $z \in \mathcal{I}$, the optimal switching control problem is to maximize the expected payoff over possible switching controls $\alpha \in \mathcal{B}$ such that $\kappa_{0}=k \in\{0,1\}$. This leads to the value functions given by

$$
\begin{aligned}
& m_{+}^{*}(z, k):=\sup _{\substack{\alpha \in \mathcal{B} \\
\kappa_{0}=k}} m_{+}(z, \alpha), \\
& m_{-}^{*}(z, k):=\sup _{\substack{\alpha \in \mathcal{B} \\
\kappa_{0}=k}} m_{-}(z, \alpha),
\end{aligned}
$$

where $m_{+}(z, \alpha)$ and $m_{-}(z, \alpha)$ are given by (19) and (20).

In fact, these two value functions (21) and (22) are essentially the same as shown in the following lemma.

LEMma 3.4. The value functions $m_{+}^{*}(z, k)$ and $m_{-}^{*}(z, k)$ in (21) and (22) satisfy, for $k \in\{0,1\}$,

$$
m_{+}^{*}(z, k)-m_{-}^{*}(z, k)=\mathbb{E}\left[\int_{0}^{\infty} \pi(t, z) d t\right] .
$$

In addition, for fixed $k \in\{0,1\}$, each switching control $\alpha \in \mathcal{B}$ that is optimal for (21) will also be optimal for (22) and vice versa.

The proof follows easily by observing that, for any control $\alpha \in \mathcal{B}$ and any fixed $z \in \mathcal{I}$

$$
m_{+}(z, \alpha)-m_{-}(z, \alpha)=\mathbb{E}\left[\int_{0}^{\infty} \pi(t, z) I_{t}+\pi(t, z)\left(1-I_{t}\right) d t\right]=\mathbb{E}\left[\int_{0}^{\infty} \pi(t, z) d t\right] .
$$

Copyright (c) by SIAM. Unauthorized reproduction of this article is prohibited. 
Next, we obtain the following lower bounds on the value functions of the switching problems, by considering the no-switching strategies $\left(\tau_{n}=\infty\right.$ for all $\left.n\right)$.

Proposition 3.5. Given $m_{+}^{*}(z, k)$ and $m_{-}^{*}(z, k)$ in (21) and (22),

$$
\begin{aligned}
m_{+}^{*}(z, 0) \geq 0, & m_{+}^{*}(z, 1) \geq \mathbb{E}\left[\int_{0}^{\infty} \pi(t, z) d t\right], \\
m_{-}^{*}(z, 0) \geq-\mathbb{E}\left[\int_{0}^{\infty} \pi(t, z) d t\right], & m_{-}^{*}(z, 1) \geq 0 .
\end{aligned}
$$

Moreover, we have the following upper bound on the value function of the singular control problem.

Proposition 3.6. Given $V(y)$ and $R(y)$ from (12) and (13) and $m_{+}^{*}(z, k)$ and $m_{-}^{*}(z, k)$ in $(21)$ and $(22)$,

$$
V(y)-R(y) \leq \int_{y}^{\infty} m_{+}^{*}(z, 0) 1_{\{z \in \mathcal{I}\}} d z+\int_{-\infty}^{y} m_{-}^{*}(z, 1) 1_{\{z \in \mathcal{I}\}} d z .
$$

Proof of Proposition 3.6. Given any integrable strategy $\left(\xi^{+}, \xi^{-}\right) \in \mathcal{A}_{y}^{\prime}$, let $(\alpha(z))_{z \in \mathcal{I}}$ be the corresponding consistent collection of switching controls. From Proposition 3.3,

$$
\begin{aligned}
J\left(y, \xi^{+}, \xi^{-}\right)-R(y) & =\int_{y}^{\infty} m_{+}(z, \alpha(z)) 1_{\{z \in \mathcal{I}\}} d z+\int_{-\infty}^{y} m_{-}(z, \alpha(z)) 1_{\{z \in \mathcal{I}\}} d z \\
& \leq \int_{y}^{\infty} m_{+}^{*}(z, 0) 1_{\{z \in \mathcal{I}\}} d z+\int_{-\infty}^{y} m_{-}^{*}(z, 1) 1_{\{z \in \mathcal{I}\}} d z
\end{aligned}
$$

since for $z>y, m_{+}(z, \alpha(z)) \leq m_{+}^{*}(z, 0)$ and for $z \leq y, m_{-}(z, \alpha(z)) \leq m_{-}^{*}(z, 1)$.

(23) follows easily by taking the supremum over all $\left(\xi^{+}, \xi^{-}\right) \in \mathcal{A}_{y}^{\prime}$.

However, the other direction of the inequality requires additional conditions to guarantee the existence of a consistent collection of optimal (or near-optimal) switching controls and that this consistent collection corresponds to an integrable singular control.

Theorem 3.7 (representation). Fix $y \in \overline{\mathcal{I}}$, and let $V(y)$ and $R(y)$ be given from (12), $m_{+}^{*}(z, k)$ and $m_{-}^{*}(z, k)$ be given by $(21)$ and $(22)$, and $\left(\hat{\xi}^{j+}, \hat{\xi}^{j-}\right) \in \mathcal{A}_{y}$ be the corresponding singular control as per Proposition 2.10. Assume there is a sequence of consistent collections of switching controls $\left(\alpha_{j}(z)\right)_{z \in \mathbb{R}}$ so that, as $j \rightarrow \infty$,

$$
\begin{gathered}
\int_{y}^{\infty} m_{+}\left(z, \alpha_{j}(z)\right) 1_{\{z \in \mathcal{I}\}} d z+\int_{-\infty}^{y} m_{-}\left(z, \alpha_{j}(z)\right) 1_{\{z \in \mathcal{I}\}} d z \\
\rightarrow \int_{y}^{\infty} m_{+}^{*}(z, 0) 1_{\{z \in \mathcal{I}\}} d z+\int_{-\infty}^{y} m_{-}^{*}(z, 1) 1_{\{z \in \mathcal{I}\}} d z
\end{gathered}
$$

Assume also that $\left(\hat{\xi}^{j+}, \hat{\xi}^{j-}\right) \in \mathcal{A}_{y}^{\prime}$ for all $j$. Then

$$
V(y)-R(y)=\int_{y}^{\infty} m_{+}^{*}(z, 0) 1_{\{z \in \mathcal{I}\}} d z+\int_{-\infty}^{y} m_{-}^{*}(z, 1) 1_{\{z \in \mathcal{I}\}} d z .
$$

Copyright $@$ by SIAM. Unauthorized reproduction of this article is prohibited. 
Proof of Theorem 3.7. Define

$$
Q(y):=\int_{y}^{\infty} m_{+}^{*}(z, 0) 1_{\{z \in \mathcal{I}\}} d z+\int_{-\infty}^{y} m_{-}^{*}(z, 1) 1_{\{z \in \mathcal{I}\}} d z .
$$

First, we treat the case where $Q(y)=\infty$. Let $\epsilon>0$ be given. From the assumption, find $j$ so large that $\frac{1}{\epsilon}<\int_{y}^{\infty} m_{+}\left(z, \alpha_{j}(z)\right) 1_{\{z \in \mathcal{I}\}} d z+\int_{-\infty}^{y} m_{-}\left(z, \alpha_{j}(z)\right) 1_{\{z \in \mathcal{I}\}} d z$. By Proposition 3.3 we have

$$
\begin{aligned}
V(y)-R(y) & \geq J\left(y, \hat{\xi}^{j+}, \hat{\xi}^{j-}\right)-R(y) \\
& =\int_{y}^{\infty} m_{+}\left(z, \alpha_{j}(z)\right) 1_{\{z \in \mathcal{I}\}} d z+\int_{-\infty}^{y} m_{-}\left(z, \alpha_{j}(z)\right) 1_{\{z \in \mathcal{I}\}} d z>\frac{1}{\epsilon} .
\end{aligned}
$$

Since $\epsilon$ is arbitrary and $R(y)$ is finite, $V(y)=\infty=Q(y)$.

Next, suppose $Q(y)<\infty$, and let $\epsilon>0$ be given. Again from the assumption, find $j$ so large that $\int_{y}^{\infty} m_{+}\left(z, \alpha_{j}(z)\right) 1_{\{z \in \mathcal{I}\}} d z+\int_{-\infty}^{y} m_{-}\left(z, \alpha_{j}(z)\right) 1_{\{z \in \mathcal{I}\}} d z>Q(y)-\epsilon$. By Propositions 3.3 and 3.6,

$$
\begin{aligned}
V(y)-R(y) & \geq J\left(y, \hat{\xi}^{+}, \hat{\xi}^{-}\right)-R(y) \\
& =\int_{y}^{\infty} m_{+}\left(z, \alpha_{j}(z)\right) 1_{\{z \in \mathcal{I}\}} d z+\int_{-\infty}^{y} m_{-}\left(z, \alpha_{j}(z)\right) 1_{\{z \in \mathcal{I}\}} d z \\
& >Q(y)-\epsilon \geq V(y)-R(y)-\epsilon .
\end{aligned}
$$

Since $\epsilon$ is arbitrary, $V(y)-R(y)=Q(y)$ as desired.

Moreover, with stronger assumptions, one can further establish the existence of an optimal control strategy, from Propositions 3.3 and 3.6.

Assumption 3.8.

1. [Existence of consistent controls]. Fix $y \in \overline{\mathcal{I}}$, and let $m_{+}^{*}(z, k)$ and $m_{-}^{*}(z, k)$ be given by (21) and (22). For almost all $z \in \mathcal{I}$, there exists an optimal admissible switching control $\alpha(z) \in \mathcal{B}$ such that

$$
\begin{aligned}
& m_{+}^{*}(z, 0)=m_{+}(z, \alpha(z)) \quad \text { for } z>y \\
& \text { and } m_{+}^{*}(z, 1)=m_{+}(z, \alpha(z)) \quad \text { for } z \leq y \text {. }
\end{aligned}
$$

Furthermore, the collection $(\alpha(z))_{z \in \mathbb{R}}$ is consistent.

2. [Integrability of singular control]. Let $\left(\hat{\xi}^{+}, \hat{\xi}^{-}\right) \in \mathcal{A}_{y}$ be the corresponding singular control as per Proposition 2.10, and then $\left(\hat{\xi}^{+}, \hat{\xi}^{-}\right) \in \mathcal{A}_{y}^{\prime}$.

Theorem 3.9 (representation and existence). Under Assumption 3.8, the representation Theorem 3.7 holds. Moreover, the strategy $\left(\hat{\xi}^{+}, \hat{\xi}^{-}\right)$is optimal.

3.2.3. Remarks on integrability of singular controls. Although establishing simpler conditions for the consistency of the switching controls requires more structure for the control problem, the equally technical integrability assumption on the singular controls can be reduced to easily verifiable ones when $\mathcal{I}$ is bounded. These extra assumptions are in line with some of those in [33].

TheOrem 3.10 (sufficient condition for integrability). Let $\mathcal{I}$ be bounded, assume 3.8.1, and let $\left(\hat{\xi}^{+}, \hat{\xi}^{-}\right)$be the corresponding singular control as per Proposition 2.10. Furthermore, suppose

Copyright (c) by SIAM. Unauthorized reproduction of this article is prohibited. 
1. $\sup _{0 \leq t \leq T} \sup _{z \in \mathcal{I}}|\Pi(\omega, t, z)|<\infty$, almost surely, for all $T>0$,

2. $\lim \sup _{T \rightarrow \infty} \mathbb{E}\left[\left|\gamma_{+}(T)\right|+\left|\gamma_{-}(T)\right|\right]<\infty$, and

3. for every strategy $\left(\xi^{+}, \xi^{-}\right) \in \mathcal{A}_{y}$, either $\left(\xi^{+}, \xi^{-}\right) \in \mathcal{A}_{y}^{\prime}$ or there exists an $\mathbb{F}$ adapted process $Z$ such that $U . \leq Z$. almost surely, $\mathbb{E}\left[\left|Z_{T}\right|\right]<\infty$ for all $T \geq 0$, and $\lim \sup _{T \rightarrow \infty} \mathbb{E}\left[Z_{T}\right]=-\infty$, where

$$
U_{T}\left(y, \xi^{+}, \xi^{-}\right):=\int_{0}^{T} \Pi\left(t, Y_{t}\right) d t-\int_{[0, T)} \gamma_{+}(t) d \xi_{t}^{+}-\int_{[0, T)} \gamma_{-}(t) d \xi_{t}^{-} .
$$

Then $\left(\hat{\xi}^{+}, \hat{\xi}^{-}\right) \in \mathcal{A}_{y}^{\prime}$. Hence Assumption 3.8 holds, yielding Theorem 3.9.

The proof is somewhat technical and thus is given in the appendix.

Note that, when $\mathcal{I}$ is unbounded, integrable consistent controls may not exist under these extra conditions. Nevertheless, we see the following.

Corollary 3.11. If $\mathcal{I}$ is unbounded, the assumptions of Theorem 3.10 yield Theorem 3.7.

Proof. Let $\mathcal{I}$ be unbounded and $(\alpha(z))_{z \in \mathcal{I}}$ be the optimal consistent collection from Assumption 3.8.1. For each $j \geq 1$, define $\alpha_{j}(z)=\alpha(z)$ for $z \in \mathcal{I} \cap(-j, j)$. For $z \notin \mathcal{I} \cap(-j, j)$, define $\alpha_{j}(z)$ to be the no action switching control (corresponding to the regime indicator function $\left.I_{t}^{j}(z)=1_{\{z \leq y\}}\right)$.

The resulting collection $\left(\alpha_{j}(z)\right)_{z \in \mathcal{I}}$ is clearly consistent, so we let $\left(\hat{\xi}^{j+}, \hat{\xi}^{j-}\right)$ be the corresponding singular controls. Furthermore, by considering the control problem restricted to $\overline{\mathcal{I}} \cap[-j, j]$, Theorem 3.10 implies that $\left(\hat{\xi}^{j+}, \hat{\xi}^{j-}\right) \in \mathcal{A}_{y}^{\prime}$. Last, by the monotone convergence theorem (since $m_{+}^{*}(z, 0)$ and $m_{-}^{*}(z, 1)$ are nonnegative), we have, as $j \rightarrow \infty$,

$$
\begin{aligned}
\int_{y}^{\infty} & m_{+}\left(z, \alpha_{j}(z)\right) 1_{\{z \in \mathcal{I}\}} d z+\int_{-\infty}^{y} m_{-}\left(z, \alpha_{j}(z)\right) 1_{\{z \in \mathcal{I}\}} d z \\
\quad & \int_{y}^{\infty} m_{+}^{*}(z, 0) 1_{\{z \in \mathcal{I} \cap(-j, j)\}} d z+\int_{-\infty}^{y} m_{-}^{*}(z, 1) 1_{\{z \in \mathcal{I} \cap(-j, j)\}} d z \\
& \rightarrow \int_{y}^{\infty} m_{+}^{*}(z, 0) 1_{\{z \in \mathcal{I}\}} d z+\int_{-\infty}^{y} m_{-}^{*}(z, 1) 1_{\{z \in \mathcal{I}\}} d z .
\end{aligned}
$$

3.3. Regularity of the value function. In this section, we provide conditions under which the value function of the switching controls is not only continuous but also continuously differentiable. As a result, we prove directly the smooth fit condition assumed a priori in [33].

Proposition 3.12. Suppose that, for some $y \in \mathcal{I}$,

$$
\lim _{z \rightarrow y} \mathbb{E}\left[\int_{0}^{\infty}|\pi(t, z)-\pi(t, y)| d t\right]=0 .
$$

Then for $k \in\{0,1\}, m_{+}^{*}(\cdot, k)$ and $m_{-}^{*}(\cdot, k)$ (from (21) and (22)) are continuous at $y$.

Proof of Proposition 3.12. Let $y \in \mathcal{I}$ and $k \in\{0,1\}$ be given, and consider any admissible strategy $\alpha \in \mathcal{B}$. By (18), (19), and (25),

$$
\lim _{z \rightarrow y}\left|m_{+}(z, \alpha)-m_{+}(y, \alpha)\right| \leq \lim _{z \rightarrow y} \mathbb{E}\left[\int_{0}^{\infty}|\pi(t, z)-\pi(t, y)| d t\right]=0 .
$$

Note that convergence to zero is uniform across all strategies $\alpha \in \mathcal{B}$.

Copyright $@$ by SIAM. Unauthorized reproduction of this article is prohibited. 
Let $\epsilon>0$ be given. There exists $\delta>0$ so that, for any strategy $\alpha \in \mathcal{B}, \mid m_{+}(z, \alpha)-$ $m_{+}(y, \alpha) \mid<\frac{\epsilon}{2}$ for all $z \in \mathcal{I}$ such that $|z-y|<\delta$.

Now there exists a strategy $\hat{\alpha} \in \mathcal{B}$ with $\kappa_{0}=k$ such that

$$
m_{+}^{*}(y, k) \leq m_{+}(y, \hat{\alpha})+\frac{\epsilon}{2} .
$$

So for all $z \in \mathcal{I}$ such that $|z-y|<\delta$,

$$
m_{+}^{*}(y, k) \leq m_{+}(y, \hat{\alpha})+\frac{\epsilon}{2} \leq m_{+}(z, \hat{\alpha})+\epsilon \leq m_{+}^{*}(z, k)+\epsilon .
$$

Furthermore, for any such $z$, there exists a switching control $\alpha_{z} \in \mathcal{B}$ with $\kappa_{0}=0$ such that

$$
m_{+}^{*}(z, k) \leq m_{+}\left(z, \alpha_{z}\right)+\frac{\epsilon}{2} \leq m_{+}\left(y, \alpha_{z}\right)+\epsilon \leq m_{+}^{*}(y, k)+\epsilon .
$$

Hence for all $z \in \mathcal{I}$ such that $|z-y|<\delta$,

$$
m_{+}^{*}(y, k)-\epsilon \leq m_{+}^{*}(z, k) \leq m_{+}^{*}(y, k)+\epsilon .
$$

Thus, $\lim _{z \rightarrow y} m_{+}^{*}(z, k)=m_{+}^{*}(y, k)$. Moreover, $\lim _{z \rightarrow y} m_{-}^{*}(z, k)=m_{-}^{*}(y, k)$ follows from Lemma 3.4 .

THEOREM 3.13 (regularity). Assume the conditions in Proposition 3.12 on an open interval $\mathcal{J} \subset \mathcal{I}$. Suppose that, on $\mathcal{J}$, the value function has the representation

$$
V(y)-R(y)=\int_{y}^{\infty} m_{+}^{*}(z, 0) 1_{\{z \in \mathcal{I}\}} d z+\int_{-\infty}^{y} m_{-}^{*}(z, 1) 1_{\{z \in \mathcal{I}\}} d z .
$$

Then $V$ is $C^{1}$ on $\mathcal{J}$, and, for any $y \in \mathcal{J}$,

$$
V^{\prime}(y)=\mathbb{E}\left[\int_{0}^{\infty} \pi(t, y) d t\right]+m_{-}^{*}(y, 1)-m_{+}^{*}(y, 0)=m_{+}^{*}(y, 1)-m_{+}^{*}(y, 0) .
$$

Proof of Theorem 3.13. By Proposition 3.12, it remains to show that $R^{\prime}(y)=$ $\mathbb{E}\left[\int_{0}^{\infty} \pi(t, y) d t\right]$. Fixing $z_{0} \in \mathcal{I}$, this follows easily from (14) and (15), and

$$
\begin{aligned}
R(y)-R\left(z_{0}\right) & =\mathbb{E}\left[\int_{0}^{\infty}\left(\Pi(t, y)-\Pi\left(t, z_{0}\right)\right) d t\right] \\
& =\mathbb{E}\left[\int_{0}^{\infty} \int_{z_{0}}^{y} \pi(t, z) d z d t\right]=\int_{z_{0}}^{y} \mathbb{E}\left[\int_{0}^{\infty} \pi(t, z) d z d t\right]
\end{aligned}
$$

Note that previous results of $[30,8,9,10]$ on the differentiability of the value function for the (ir)reversible investment problem are special cases of ours. Another major difference is that the derivative in their work is in terms of the value of a Dynkin game, whereas the derivative here is the difference between the value functions of an optimal switching problem.

In the remainder of this section, we shall show that, under very mild assumptions, the value of a Dynkin game exists and is equal to the difference of the value functions for the optimal switching problem defined by (19) and (20); thereby we demonstrate that optimal switching problems provide a "missing link" between Dynkin games and singular control problems.

For simplicity, we consider an infinite-horizon Dynkin game with no terminal payoff. With a slight modification, our arguments can be adapted for the finitehorizon case. 
3.3.1. Dynkin games. A Dynkin game is a game of timing between two players, whom we call MAX and MIN, following [10]. We fix some level $z \in \mathcal{I}$. While the game is in progress, MIN pays MAX at rate $\pi(t, z)$, and the game ends when one player chooses to stop. Thus, MAX and MIN each choose strategies on when to exit the game (the stopping times $\sigma_{-}$and $\sigma_{+}$, respectively). The player to exit first pays an amount to her opponent equal to $\gamma_{-}\left(\sigma_{-}\right)$if MAX exits first and $\gamma_{+}\left(\sigma_{+}\right)$if MIN exits first. If both players exit at the same time, we treat it as though MIN exited first. Furthermore, each player may choose never to exit, i.e., $\sigma=\infty$. MAX chooses her strategy $\sigma_{-}$to maximize her payoff, and MIN chooses $\sigma_{+}$in order to minimize MAX's payoff.

This game is formally described below. To ensure that the payoff of the game is well-defined, we assume in this section that, for every stopping time $\sigma, \mathbb{E}\left[\left|\gamma_{-}(\sigma)\right|\right]<\infty$ and $\mathbb{E}\left[\left|\gamma_{+}(\sigma)\right|\right]<\infty$.

Definition 3.14. Given $z \in \mathcal{I}$ and $\mathbb{F}$-stopping times $\sigma_{-}$and $\sigma_{+}$, the payoff of the Dynkin game is

$$
D\left(\sigma_{-}, \sigma_{+} ; z\right)=\int_{0}^{\sigma_{-} \wedge \sigma_{+}} \pi(t, z) d t+\gamma_{+}\left(\sigma_{+}\right) 1_{\left\{\sigma_{+} \leq \sigma_{-}\right\}}-\gamma_{-}\left(\sigma_{-}\right) 1_{\left\{\sigma_{-}<\sigma_{+}\right\}} .
$$

The game has a value if

$$
\sup _{\sigma_{-}} \inf _{\sigma_{+}} \mathbb{E}\left[D\left(\sigma_{-}, \sigma_{+} ; z\right)\right]=\inf _{\sigma_{+}} \sup _{\sigma_{-}} \mathbb{E}\left[D\left(\sigma_{-}, \sigma_{+} ; z\right)\right]
$$

It is easy to see that $\sup _{\sigma_{-}} \inf _{\sigma_{+}} \mathbb{E}\left[D\left(\sigma_{-}, \sigma_{+} ; z\right)\right] \leq \inf _{\sigma_{+}} \sup _{\sigma_{-}} \mathbb{E}\left[D\left(\sigma_{-}, \sigma_{+} ; z\right)\right]$. Moreover, we have the following theorem.

TheOREM 3.15. Given any $z \in \mathcal{I}$ such that conditions (16) and (17) hold, the value of the Dynkin game exists and is equal to

$$
m_{+}^{*}(z, 1)-m_{+}^{*}(z, 0)=\sup _{\sigma_{-}} \inf _{\sigma_{+}} \mathbb{E}\left[D\left(\sigma_{-}, \sigma_{+} ; z\right)\right]=\inf _{\sigma_{+}} \sup _{\sigma_{-}} \mathbb{E}\left[D\left(\sigma_{-}, \sigma_{+} ; z\right)\right]
$$

Proof of Theorem 3.15. It suffices to show $m_{+}^{*}(z, 1)-m_{+}^{*}(z, 0) \leq \sup _{\sigma_{-}} \inf _{\sigma_{+}}$ $\mathbb{E}\left[D\left(\sigma_{-}, \sigma_{+} ; z\right)\right]$, since it follows similarly for $m_{+}^{*}(z, 1)-m_{+}^{*}(z, 0) \geq \inf _{\sigma_{+}} \sup _{\sigma_{-}}$ $\mathbb{E}\left[D\left(\sigma_{-}, \sigma_{+} ; z\right)\right]$.

Note that $m_{+}^{*}(z, 1)-m_{+}^{*}(z, 0) \leq \sup _{\sigma_{-}} \inf _{\sigma_{+}} \mathbb{E}\left[D\left(\sigma_{-}, \sigma_{+} ; z\right)\right]$ if and only if, for all $\epsilon>0$, there exists $\hat{\sigma}_{-}$such that, for all $\sigma_{+}, \mathbb{E}\left[D\left(\hat{\sigma}_{-}, \sigma_{+} ; z\right)\right]+\epsilon \geq m_{+}^{*}(z, 1)-m_{+}^{*}(z, 0)$.

Let $\epsilon>0$ be given, let $\alpha^{1} \in \mathcal{B}$ be a switching control with $\kappa_{0}=1$ such that

$$
m_{+}\left(z, \alpha^{1}\right)+\epsilon \geq m_{+}^{*}(z, 1)
$$

and define $\hat{\sigma}_{-}=\tau_{1}=\inf \left\{t: I_{t}^{1}=0\right\}$.

Let $\sigma_{+}$be an arbitrary stopping time, and define $\alpha^{0}$ by taking $I_{t}^{0}=0$ for $t \leq$ $\hat{\sigma}_{-} \wedge \sigma_{+}$and $I_{t}^{0}=I_{t}^{1}$ for $t>\hat{\sigma}_{-} \wedge \sigma_{+}$. Thus $I^{0}$ is a regime indicator function, and hence $\alpha^{0}$ is an admissible switching control. In fact, since $\alpha^{1} \in \mathcal{B}$, we also have $\alpha^{0} \in \mathcal{B}$. 
Thus, for any $\sigma_{+}$,

$$
\begin{aligned}
& m_{+}^{*}(z, 1)- m_{+}^{*}(z, 0) \leq m_{+}^{*}(z, 1)-m_{+}\left(z, \alpha^{0}\right) \leq m_{+}\left(z, \alpha^{1}\right)-m_{+}\left(z, \alpha^{0}\right)+\epsilon \\
&= \mathbb{E}\left[\int_{0}^{\infty} \pi(t, z)\left(I_{t}^{1}-I_{t}^{0}\right) d t+\gamma_{+}\left(\sigma_{+}\right) 1_{\left\{\sigma_{+}<\hat{\sigma}_{-}\right\}}-\gamma_{-}\left(\hat{\sigma}_{-}\right) 1_{\left\{\hat{\sigma}_{-} \leq \sigma_{+}\right\}}\right]+\epsilon \\
&= \mathbb{E}\left[\int_{0}^{\hat{\sigma}_{-} \wedge \sigma_{+}} \pi(t, z) d t+\gamma_{+}\left(\sigma_{+}\right) 1_{\left\{\sigma_{+}<\hat{\sigma}_{-}\right\}}-\gamma_{-}\left(\hat{\sigma}_{-}\right) 1_{\left\{\hat{\sigma}_{-} \leq \sigma_{+}\right\}}\right]+\epsilon \\
&= \mathbb{E}\left[\int_{0}^{\hat{\sigma}_{-} \wedge \sigma_{+}} \pi(t, z) d t+\gamma_{+}\left(\sigma_{+}\right) 1_{\left\{\sigma_{+}<\hat{\sigma}_{-}\right\}}\right. \\
&\left.\quad-\gamma_{-}\left(\hat{\sigma}_{-}\right) 1_{\left\{\hat{\sigma}_{-}<\sigma_{+}\right\}}-\gamma_{-}\left(\sigma_{+}\right) 1_{\left\{\hat{\sigma}_{-}=\sigma_{+}\right\}}\right]+\epsilon \\
& \leq \mathbb{E}\left[\int_{0}^{\hat{\sigma}_{-} \wedge \sigma_{+}} \pi(t, z) d t+\gamma_{+}\left(\sigma_{+}\right) 1_{\left\{\sigma_{+} \leq \hat{\sigma}_{-}\right\}}-\gamma_{-}\left(\hat{\sigma}_{-}\right) 1_{\left\{\hat{\sigma}_{-}<\sigma_{+}\right\}}\right]+\epsilon \\
&=\mathbb{E}\left[D\left(\hat{\sigma}_{-}, \sigma_{+} ; z\right)\right]+\epsilon,
\end{aligned}
$$

where the last inequality follows from (17). Thus,

$$
m_{+}^{*}(z, 1)-m_{+}^{*}(z, 0) \leq \sup _{\sigma_{-}} \inf _{\sigma_{+}} \mathbb{E}\left[D\left(\sigma_{-}, \sigma_{+} ; z\right)\right] .
$$

Furthermore, we see the following.

Corollary 3.16. If (16) and (17) hold and $\pi(t, z)$ is decreasing in $z$, then $m_{+}^{*}(z, 1)-m_{+}^{*}(z, 0)$ is decreasing in $z$.

That is, when the marginal payoff is decreasing in the capacity level $z$, the added benefit of being invested in the project at level $z$ is also decreasing in $z$. The economic interpretation is that there are decreasing returns to scale.

3.4. Examples with explicit solutions. We now illustrate how our methodology can be used to solve a reversible investment problem with a Cobb-Douglas production function. This is a special case of the problem solved in [33]. Note that, although our method can handle the general problem in [33] among others, we nevertheless have selected this simple case to illustrate our techniques: Unlike [33], we solve without assuming a priori the continuous differentiability of the value function or any assumptions on the structure of the switching regions.

Singular control problem.

$$
V(x, y):=\sup _{\left(\xi^{+}, \xi^{-}\right) \in \mathcal{A}_{y}^{\prime}} \mathbb{E}\left[\int_{0}^{\infty} \Pi\left(t, Y_{t}\right) d t-\int_{[0, \infty)} \gamma_{+}(t) d \xi_{t}^{+}-\int_{[0, \infty)} \gamma_{-}(t) d \xi_{t}^{-}\right]
$$

Copyright $@$ by SIAM. Unauthorized reproduction of this article is prohibited. 
subject to

$$
\begin{array}{ll} 
& \xi^{+}(0)=\xi^{-}(0):=0 \\
& Y_{t}:=y+\xi_{t}^{+}-\xi_{t}^{-} \in \overline{\mathcal{I}} \quad \forall t \in[0, \infty), \\
& \Pi(\omega, t, y):=e^{-\rho t}\left(X_{t}^{x}(\omega)\right)^{\lambda} y^{\beta}, \\
& d X_{t}^{x}:=b X_{t}^{x} d t+\sqrt{2} \sigma X_{t}^{x} d W_{t}, \quad X_{0}:=x>0, \\
\text { and } \quad \gamma_{+}(t):=e^{-\rho t} K_{1}, \gamma_{-}(t):=e^{-\rho t} K_{0} .
\end{array}
$$

For simplicity, we assume $\overline{\mathcal{I}}=[A, B] \subset[0, \infty)$ is a bounded interval, $\rho>0, \lambda \in(0, n)$, $\beta \in(0,1]$, and $K_{0}<0, K_{1}>0$, with $K_{0}+K_{1}>0$. Here $n=\frac{-\left(b-\sigma^{2}\right)+\sqrt{\left(b-\sigma^{2}\right)^{2}+4 \sigma^{2} \rho}}{2 \sigma^{2}}$. This formulation is from [33].

This problem is solved in several steps.

Step 1: Corresponding optimal switching problem. First, one can check that standing assumptions A1, A2, and A3 and the assumptions in Theorem 3.10 hold for this problem, with

$$
\pi(\omega, t, y)=\beta e^{-\rho t}\left(X_{t}^{x}(\omega)\right)^{\lambda} y^{-(1-\beta)}
$$

Also, $R(x, y)$ is differentiable in $y$, so that

$$
R(x, y)=\mathbb{E}\left[\int_{0}^{\infty} \Pi(t, y) d t\right]=\frac{-x^{\lambda} y^{\beta}}{\sigma^{2} \lambda^{2}+\left(b-\sigma^{2}\right) \lambda-\rho}
$$

and

$$
r(x, y)=R_{y}(x, y)=\mathbb{E}\left[\int_{0}^{\infty} \pi(t, y) d t\right]=\frac{-\beta x^{\lambda} y^{-(1-\beta)}}{\sigma^{2} \lambda^{2}+\left(b-\sigma^{2}\right) \lambda-\rho} .
$$

By Lemma 3.4, it suffices to solve for the optimal switching problem defined by

$$
v_{k}(x, z):=m_{+}^{*}(x, z, k)=\sup _{\substack{\alpha \in \mathcal{B} \\ \kappa_{0}=k}} \mathbb{E}\left[\int_{0}^{\infty} e^{-\rho t} \beta z^{-(1-\beta)}\left(X_{t}^{x}\right)^{\lambda} I_{t} d t-\sum_{n=1}^{\infty} e^{-\rho \tau_{n}} K_{\kappa_{n}}\right] \text {. }
$$

Step 2: Consistent collection of optimal switching controls. Applying the regularity results of [32] for switching controls to problem (27), we see that, for any given $z \in(A, B)$ and $k \in\{0,1\}$, the value function $v_{k}(\cdot, z)$ is continuously differentiable. Moreover, for each $z \in(A, B)$, an optimal switching control exists and can be described in terms of the switching regions: For each $z \in(A, B)$, there exist $0<F(z)<G(z)<\infty$ such that it is optimal to switch from regime 0 to regime 1 (to invest in the project at level $z$ ) when $X_{t}^{x} \in[G(z), \infty)$ and to switch from regime 1 to regime 0 (disinvest at level $z$ ) when $X_{t}^{x} \in[0, F(z)]$.

Therefore, given any initial value $y \in[A, B]$ for the singular control problem, a collection of optimal switching controls can be defined as follows.

For $(x, z) \in \mathcal{X} \times(A, B)$, define the switching control $\hat{\alpha}(x, z)=\left(\hat{\tau}_{n}, \hat{\kappa}_{n}\right)_{n \geq 0}$, starting from $\hat{\tau}_{0}=0$ and $\kappa_{0}=1_{\{z \leq y\}}$ by setting $\hat{\kappa}_{n}:=1-\kappa_{n-1}$ for all $n \geq 1$ and

- if $\kappa_{n-1}=0, \hat{\tau}_{n}:=\inf \left\{t>\tau_{n-1}: X_{t}^{x} \geq G(z)\right\}$, or 
- if $\kappa_{n-1}=1, \hat{\tau}_{n}:=\inf \left\{t>\tau_{n-1}: X_{t}^{x} \leq F(z)\right\}$.

Moreover, by the regularity of the value functions, we solve for $F(z)$ and $G(z)$ explicitly in our case, obtaining $F(z)=\kappa z^{\frac{1-\beta}{\lambda}}$ and $G(z)=\nu z^{\frac{1-\beta}{\lambda}}$, where $\kappa$ and $\nu$ are unique solutions to

$$
\begin{gathered}
\frac{\beta}{\lambda-m}\left[\nu^{\lambda-m}-\kappa^{\lambda-m}\right]=-\frac{\rho}{m}\left[K_{1} \nu^{-m}+K_{0} \kappa^{-m}\right], \\
\frac{\beta}{n-\lambda}\left[\nu^{\lambda-n}-\kappa^{\lambda-n}\right]=\frac{\rho}{n}\left[K_{1} \nu^{-n}+K_{0} \kappa^{-n}\right] .
\end{gathered}
$$

Here $m<0<n$, and $n, m=\frac{-\left(b-\sigma^{2}\right) \pm \sqrt{\left(b-\sigma^{2}\right)^{2}+4 \sigma^{2} r}}{2 \sigma^{2}}$.

Finally, by checking the appropriate integrability conditions, and by noting that $F$ and $G$ are increasing in $z$, it is not hard to verify that the above collection of optimal switching controls is consistent. (See Figure 1.)

Step 3: Optimal singular control and value functions. By Proposition 2.10, this consistent collection of optimal switching control corresponds to an admissible singular control $\left(\hat{\xi}^{+}, \hat{\xi}^{-}\right) \in \mathcal{A}_{y}$. Moreover, since $\mathcal{I}$ is bounded, it is integrable following Theorem 3.10.

Put together, the investment region is given by $\{(x, z): x \geq G(z)\}$ and the disinvestment region by $\{(x, z): x \leq F(z)\}$. $Y_{t}$ is constant when $\left(X_{t}, Y_{t}\right)$ is in the wait region, given by $\{(x, z): F(z)<x<G(z)\}$. If $(x, y)$ is in the investment (or disinvestment) region, then a jump is exerted at time zero to make $Y_{0+}=G^{-1}(x)$ (or $\left.Y_{0+}=F^{-1}(x)\right)$.

(1) For fixed $z_{0}$ switching control

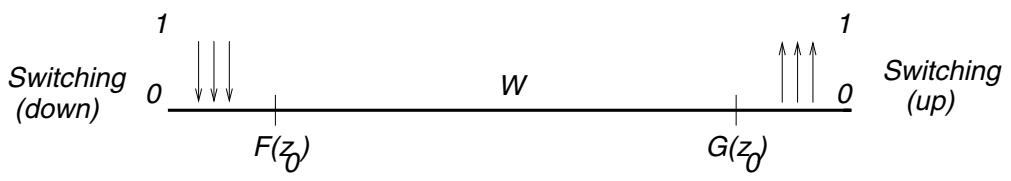

(2) For general z, consistent switching controls

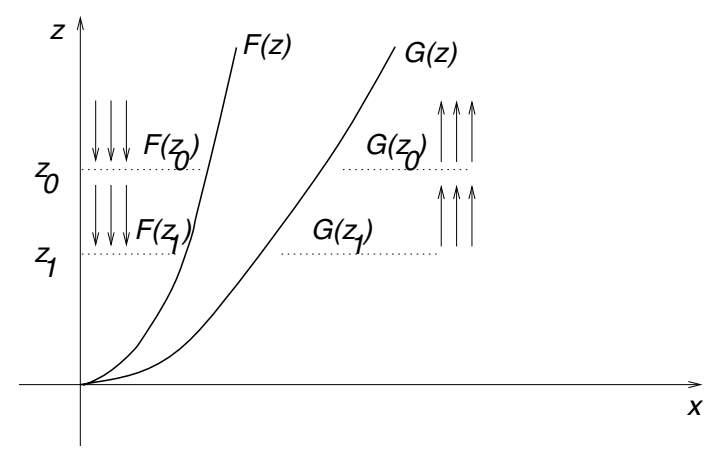

FIG. 1. Illustration of optimal consistent switching control from optimal singular control.

Copyright $@$ by SIAM. Unauthorized reproduction of this article is prohibited. 
Finally, by Lemma 3.4 and Theorem 3.9 the value function has the following representation:

$$
\begin{aligned}
V(x, y) & =R(x, y)+\int_{y}^{B} v_{0}(x, z) d z+\int_{A}^{y}\left(v_{1}(x, z)-r(x, z)\right) d z \\
& =R(x, A)+\int_{y}^{B} v_{0}(x, z) d z+\int_{A}^{y} v_{1}(x, z) d z
\end{aligned}
$$

where $v_{0}$ and $v_{1}$ are given by [32, Theorem 4.2.3]

$$
\begin{aligned}
& v_{0}(x, z)= \begin{cases}A(z) x^{n}, & x<G(z), \\
B(z) x^{m}+r(x, z)-K_{1}, & x \geq G(z),\end{cases} \\
& v_{1}(x, z)= \begin{cases}A(z) x^{n}-K_{0}, & x \leq F(z), \\
B(z) x^{m}+r(x, z), & x>F(z),\end{cases}
\end{aligned}
$$

where $A(z)=\frac{\nu^{-n}}{n-m}\left(\frac{\beta \nu^{\lambda}}{\sigma^{2}(n-\lambda)}+m K_{1}\right) z^{\frac{-n(1-\beta)}{\lambda}}, B(z)=-\frac{\kappa^{-m}}{n-m}\left(\frac{\beta \kappa^{\lambda}}{\sigma^{2}(\lambda-m)}+n K_{0}\right)$

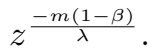

The above results are consistent with equations (117)-(120) and Remark 4 in [33].

4. Conclusions. This paper builds a generic connection between singular controls of finite variation and sequential optimal stopping problems. This correspondence is independent of any particular formulation of control problems and provides a novel method for solving explicitly high-dimensional singular control problems where randomness may not be necessarily captured by a diffusion and where payoff functions can be nonsmooth. It also enables us to derive sufficient conditions for the existence of optimal controls, for the smooth fit principle, and for the regularity of value functions. Consequently, this regularity result links singular controls and Dynkin games through sequential optimal stopping problems.

Appendix A. Proof of Theorem 3.10. The proof is built on the following proposition.

Proposition A.1. Under Assumption 3.8.1, for almost all $z \in \mathcal{I}$ and any $T>0$,

$$
\mathbb{E}\left[u_{T}(z, \alpha(z))\right] \geq\left\{\begin{array}{l}
-\mathbb{E}\left[\gamma_{+}(T) I_{T+}(z)\right] \quad \text { when } z>y, \\
-\mathbb{E}\left[\gamma_{-}(T)\left(1-I_{T+}(z)\right)\right] \quad \text { when } z \leq y,
\end{array}\right.
$$

where $\alpha(z) \in \mathcal{B}$ is given by Assumption 3.8.1 and

$u_{T}(z, \alpha(z))=\left\{\begin{array}{l}\int_{0}^{T} \pi(t, z) I_{t}(z) d t-\sum_{n=1}^{\infty} \gamma\left(\tau_{n}(z), \kappa_{n}(z)\right) 1_{\left\{\tau_{n}(z)<T\right\}}, \quad z>y, \\ \int_{0}^{T}-\pi(t, z)\left(1-I_{t}(z)\right) d t-\sum_{n=1}^{\infty} \gamma\left(\tau_{n}(z), \kappa_{n}(z)\right) 1_{\left\{\tau_{n}(z)<T\right\}}, \quad z \leq y .\end{array}\right.$

Proof. By Assumption 3.8.1, $\alpha(z)$ is an optimal admissible switching control for almost all $z \in \mathcal{I}$. Fix such a $z \in \mathcal{I}$. Consider the admissible switching control $\tilde{\alpha}_{T}(z)$ defined by the regime indicator function

$$
\tilde{I}_{t}(z)=I_{0}(z) 1_{\{t \leq T\}}+I_{t}(z) 1_{\{t>T\}} .
$$

Assume for now $z>y$. In the new switching control we have defined, we may have to switch at time $T$ from regime $\kappa_{0}=0$ to regime $I_{T+}(z)$, if $I_{T+}(z)=1$. Hence, 
the cost of the possible switch at $T$ is given by $-\gamma_{+}(T) I_{T+}(z)$. After time $T$, the switching costs are the same for both strategies.

Since the switching control $\alpha(z) \in \mathcal{B}$ is optimal, $m_{+}(z, \alpha(z))-m_{+}\left(z, \tilde{\alpha}_{T}(z)\right) \geq 0$. This means that

$$
\begin{aligned}
0 \leq & m_{+}(z, \alpha(z))-m_{+}(z, \tilde{\alpha}(z))=\mathbb{E}\left[\int_{0}^{\infty} \pi(t, z) I_{t}(z) d t-\sum_{n=1}^{\infty} \gamma\left(\tau_{n}(z), \kappa_{n}(z)\right)\right] \\
& -\mathbb{E}\left[\int_{0}^{\infty} \pi(t, z) \tilde{I}_{t}(z) d t-\sum_{n=1}^{\infty} \gamma\left(\tilde{\tau}_{n}(z), \tilde{\kappa}_{n}(z)\right)\right] \\
= & \mathbb{E}\left[\int_{0}^{\infty} \pi(t, z) I_{t}(z) d t-\sum_{n=1}^{\infty} \gamma\left(\tau_{n}(z), \kappa_{n}(z)\right)\right] \\
& -\mathbb{E}\left[\int_{T}^{\infty} \pi(t, z) I_{t}(z) d t-\gamma_{+}(T) I_{T+}(z)-\sum_{n=1}^{\infty} \gamma\left(\tau_{n}(z), \kappa_{n}(z)\right) 1_{\left\{T<\tau_{n}(z)\right\}}\right] \\
= & \mathbb{E}\left[\gamma_{+}(T) I_{T+}(z)\right]+\mathbb{E}\left[\int_{0}^{T} \pi(t, z) I_{t}(z) d t-\sum_{n=1}^{\infty} \gamma\left(\tau_{n}(z), \kappa_{n}(z)\right) 1_{\left\{\tau_{n}(z)<T\right\}}\right] .
\end{aligned}
$$

Thus, by Assumption 3.8, $\mathbb{E}\left[u_{T}(z)\right] \geq-\mathbb{E}\left[\gamma_{+}(T) I_{T+}(z)\right]$ for almost all $z>y$.

Similarly, for almost all $z \leq y$,

$$
\begin{aligned}
0 \leq & \mathbb{E}\left[\gamma_{-}(T)\left(1-I_{T+}(z)\right)\right] \\
& +\mathbb{E}\left[\int_{0}^{T}-\pi(t, z)\left(1-I_{t}(z)\right) d t-\sum_{n=1}^{\infty} \gamma\left(\tau_{n}(z), \kappa_{n}(z)\right) 1_{\left\{\tau_{n}(z)<T\right\}}\right] .
\end{aligned}
$$

Hence the claim.

Proof of Theorem 3.10. Suppose $\mathcal{I}$ is bounded, and let $(\alpha(z))_{z \in \mathcal{I}}$ be the collection of optimal, consistent switching controls given by Assumption 3.8.1 and $\left(\hat{\xi}^{+}, \hat{\xi}^{-}\right) \in \mathcal{A}_{y}$ be the corresponding singular control. The idea of the proof is to show that, for any $\mathbb{F}$-adapted process $Z$ with $U_{T} \leq Z_{T}$ and $\mathbb{E}\left[\left|Z_{T}\right|\right]<\infty$ almost surely for each $T>0$, we have $\lim \sup _{T \rightarrow \infty} \mathbb{E}\left[Z_{T}\right]>-\infty$. It then follows from the assumptions of the theorem that $\left(\hat{\xi}^{+}, \hat{\xi}^{-}\right) \in \mathcal{A}_{y}^{\prime}$.

By the assumptions of the theorem, for any fixed $T<\infty,\left|U_{T}\left(y, \hat{\xi}^{+}, \hat{\xi}^{-}\right)\right|<$ $\infty$ almost surely. Furthermore, by applying the same arguments as in the proof of Proposition 3.3 we get

$$
U_{T}\left(y, \hat{\xi}^{+}, \hat{\xi}^{-}\right)-\int_{0}^{T} \Pi(t, y) d t=\int_{\mathcal{I}} u_{T}(z) d z,
$$

where $u_{T}$ is defined in Proposition A.1.

Let $Z$ be any $\mathbb{F}$-adapted process with $U_{T} \leq Z_{T}$ and $\mathbb{E}\left[\left|Z_{T}\right|\right]<\infty$ almost surely for each $T>0$. Thus,

$$
Z_{T} \geq U_{T}\left(y, \hat{\xi}^{+}, \hat{\xi}^{-}\right)=\int_{\mathcal{I}} u_{T}(z, \alpha(z)) d z .
$$

Copyright $@$ by SIAM. Unauthorized reproduction of this article is prohibited. 
Since $\mathcal{I}$ is bounded and $\left|u_{T}\right|$ has finite expectation, $\int_{\mathcal{I}} \mathbb{E}\left[\left|u_{T}(z, \alpha(z))\right|\right] d z<\infty$. Hence by Fubini's theorem and Proposition A.1,

$$
\begin{aligned}
\mathbb{E}\left[Z_{T}\right] \geq & \mathbb{E}\left[\int_{\mathcal{I}} u_{T}(z, \alpha(z)) d z+\int_{0}^{T} \Pi(t, y) d t\right] \\
\geq & \int_{\mathcal{I}} \mathbb{E}\left[u_{T}(z, \alpha(z))\right] d z-\mathbb{E}\left[\int_{0}^{\infty}|\Pi(t, y)| d t\right] \\
\geq & -\int_{y}^{\infty} \mathbb{E}\left[\gamma_{+}(T) I_{T+}(z)\right] 1_{\{z \in \mathcal{I}\}} d z-\int_{-\infty}^{y} \mathbb{E}\left[\gamma_{-}(T)\left(1-I_{T+}(z)\right)\right] 1_{\{z \in \mathcal{I}\}} d z \\
& -\mathbb{E}\left[\int_{0}^{\infty}|\Pi(t, y)| d t\right] \\
= & \mathbb{E}\left[-\gamma_{+}(T)\left[Y_{T+}-y\right]^{+}-\gamma_{-}(T)\left[Y_{T+}-y\right]^{-}\right]-\mathbb{E}\left[\int_{0}^{\infty}|\Pi(t, y)| d t\right] \\
\geq & -C \mathbb{E}\left[\left|\gamma_{+}(T)\right|+\left|\gamma_{-}(T)\right|\right]-\mathbb{E}\left[\int_{0}^{\infty}|\Pi(t, y)| d t\right],
\end{aligned}
$$

where $C=\sup \mathcal{I}-\inf \mathcal{I}<\infty$.

Thus, by (15) and the assumptions of the theorem, $\lim \sup _{T \rightarrow \infty} \mathbb{E}\left[Z_{T}\right]>$ $-\infty$.

Acknowledgments. The authors thank the Associate Editor and the two anonymous referees for their constructive and detailed suggestions and remarks, which led to a substantial improvement of the paper. Generous support from the OpenLink Fund at the Coleman Fung Risk Management Center at UC Berkeley is gratefully acknowledged.

\section{REFERENCES}

[1] A. B. Abel And J. C. EBerly, An exact solution for the investment and value of a firm facing uncertainty, adjustment costs, and irreversibility, J. Econom. Dynam. Control, 21 (1997), pp. 831-852.

[2] F. M. Baldursson and I. Karatzas, Irreversible investment and industry equilibrium, Finance Stoch., 1 (1997), pp. 69-89.

[3] P. BANK, Optimal control under a dynamic fuel constraint, SIAM J. Control Optim., 44 (2005), pp. 1529-1541.

[4] J. Bather And H. Chernoff, Sequential decisions in the control of a spaceship, in Proceedings of the Fifth Berkeley Symposium on Mathematical Statistics and Probability (Berkeley, California, 1965/66), Vol. III: Physical Sciences, University of California Press, Berkeley, CA, 1967, pp. 181-207.

[5] J. Bather and H. Chernoff, Sequential decisions in the control of a spaceship (finite fuel), J. Appl. Probab., 4 (1967), pp. 584-604.

[6] V. E. Beneš, L. A. Shepp, and H. S. Witsenhausen, Some solvable stochastic control problems, Stochastics, 4 (1980), pp. 39-83.

[7] A. Bensoussan and J.-L. Lions, Impulse Control and Quasivariational Inequalities, GauthierVillars, Montrouge, 1984.

[8] F. Boetius, Bounded variation singular stochastic control and associated Dynkin game, in Mathematical Finance (Konstanz, 2000), Trends Math., Birkhäuser, Basel, 2001, pp. 111120 .

[9] F. Boetius, Singular Stochastic Control and its Relations to Dynkin Game and Entry-Exit Problems, Ph.D. dissertation, Universität Konstanz, Konstanz, Germany, 2003.

Copyright $@$ by SIAM. Unauthorized reproduction of this article is prohibited. 
[10] F. Boetius, Bounded variation singular stochastic control and Dynkin game, SIAM J. Control Optim., 44 (2005), pp. 1289-1321.

[11] F. Boetius And M. Kohlmann, Connections between optimal stopping and singular stochastic control, Stochastic Process. Appl., 77 (1998), pp. 253-281.

[12] K. A. BREKKe AND B. ØKSENDAL, Optimal switching in an economic activity under uncertainty, SIAM J. Control Optim., 32 (1994), pp. 1021-1036.

[13] M. B. Chiarolla and U. G. Haussmann, Explicit solution of a stochastic, irreversible investment problem and its moving threshold, Math. Oper. Res., 30 (2005), pp. 91-108.

[14] M. H. A. Davis, M. A. H. Dempster, S. P. Sethi, and D. Vermes, Optimal capacity expansion under uncertainty, Adv. in Appl. Probab., 19 (1987), pp. 156-176.

[15] A. K. Dixit And R. S. Pindyck, Investment under Uncertainty, Princeton University Press, Princeton, NJ, 1994.

[16] J. K. Duckworth And M. Zervos, An investment model with entry and exit decisions, J. Appl. Probab., 37 (2000), pp. 547-559.

[17] N. El Karoui And I. Karatzas, Probabilistic aspects of finite-fuel, reflected follower problems, Acta Appl. Math., 11 (1988), pp. 223-258.

[18] N. El Karoui AND I. Karatzas, Integration of the optimal risk in a stopping problem with absorption, in Séminaire de Probabilités, XXIII, Lecture Notes in Math. 1372, Springer, Berlin, 1989, pp. 405-420.

[19] N. El Karoui and I. Karatzas, A new approach to the Skorohod problem, and its applications, Stoch. Stoch. Rep., 34 (1991), pp. 57-82.

[20] L. C. Evans and R. F. Gariepy, Measure Theory and Fine Properties of Functions, Stud. Adv. Math., CRC Press, Boca Raton, FL, 1992.

[21] X. Guo And H. Pham, Optimal partially reversible investment with entry decision and general production function, Stochastic Process. Appl., 115 (2005), pp. 705-736.

[22] S. HAMAdÈne AND M. HASSANI, BSDEs with two reflecting barriers driven by a Brownian and a Poisson noise and related Dynkin game, Electron. J. Probab., 11 (2006), pp. 121-145.

[23] S. Hamadène and M. Jeanblanc, On the starting and stopping problem: Application in reversible investments, Math. Oper. Res., 32 (2007), pp. 182-192.

[24] J. M. HARrison And M. I. TAKsar, Instantaneous control of Brownian motion, Math. Oper. Res., 8 (1983), pp. 439-453.

[25] I. Karatzas, A class of singular stochastic control problems, Adv. in Appl. Probab., 15 (1983), pp. 225-254.

[26] I. Karatzas, Probabilistic aspects of finite-fuel stochastic control, Proc. Natl. Acad. Sci. USA, 82 (1985), pp. 5579-5581.

[27] I. Karatzas and S. E. Shreve, Connections between optimal stopping and singular stochastic control I. Monotone follower problems, SIAM J. Control Optim., 22 (1984), pp. 856-877.

[28] I. Karatzas and S. E. Shreve, Connections between optimal stopping and singular stochastic control II. Reflected follower problems, SIAM J. Control Optim., 23 (1985), pp. 433-451.

[29] I. Karatzas and S. E. Shreve, Equivalent models for finite-fuel stochastic control, Stochastics, 18 (1986), pp. 245-276.

[30] I. Karatzas AND H. WANG, Connections between bounded variation control and Dynkin games, in Optimal Control and Partial Differential Equations (volume in honor of A. Bensoussan), J. Menaldi, E. Rofman, and A. Sulem, eds., Institute of Science Press, Amsterdam, 2001, pp. 363-373.

[31] Т. Ø. KoBila, A class of solvable stochastic investment problems involving singular controls, Stoch. Stoch. Rep., 43 (1993), pp. 29-63.

[32] V. Ly Vath And H. Pham, Explicit solution to an optimal switching problem in the two-regime case, SIAM J. Control Optim., 46 (2007), pp. 395-426.

[33] A. Merhi and M. Zervos, A model for reversible investment capacity expansion, SIAM J. Control Optim., 46 (2007), pp. 839-876.

[34] A. ØKsendal, Irreversible investment problems, Finance Stoch., 4 (2000), pp. 223-250.

[35] J. A. Scheinkman and T. Zariphopoulou, Optimal environmental management in the presence of irreversibilities, J. Econom. Theory, 96 (2001), pp. 180-207.

[36] H. WANG, Capacity expansion with exponential jump diffusion processes, Stoch. Stoch. Rep., 75 (2003), pp. 259-274.

Copyright (c) by SIAM. Unauthorized reproduction of this article is prohibited. 